\title{
Decitabine-based chemotherapy followed by haploidentical lymphocyte infusion improves the effectiveness in elderly patients with acute myeloid leukemia
}

\author{
Yu Jing ${ }^{1, *}$, Xiangshu Jin ${ }^{1, *}$, Lixin Wang ${ }^{1,2, *}$, Liping Dou ${ }^{1}$, Quanshun Wang ${ }^{1}$, Yushi \\ Yao ${ }^{1}$, Shimei Lian ${ }^{3}$, Jihao Zhou ${ }^{1}$, Haiyan Zhu ${ }^{1}$, Zilong Yao', Lijun Gao', Lili Wang ${ }^{1}$, \\ Yonghui $\mathrm{Li}^{1}$, Xuefeng $\mathrm{Bai}^{4}$, Meiyun Fang ${ }^{5}$ and $\mathrm{Li} \mathrm{Yu}^{1}$ \\ ${ }^{1}$ Department of Hematology, Chinese PLA General Hospital, Beijing, China \\ 2 Department of Hematology, Navy General Hospital of PLA, Beijing, China \\ ${ }^{3}$ Department of Hematology, Dalian Municipal Central Hospital, Affiliated Hospital of Dalian Medical University, Dalian, China \\ ${ }^{4}$ Department of Pathology and Comprehensive Cancer Center, The Ohio State University Medical Center, Columbus, OH, USA \\ ${ }^{5}$ Department of Hematology, The First Affiliated Hospital of Dalian Medical University, Dalian, China \\ * These authors have contributed equally to this work \\ Correspondence to: Li Yu, email: chunhuiliyu@yahoo.com
}

Meiyun Fang, email: Fangmeiyun@aliyun.com

Keywords: decitabine, haploidentical lymphocyte infusion, Induction therapy, acute myeloid leukemia

Received: November 01,2015 Accepted: July 16,2016 Published: August 10, 2016

Copyright: Jing et al. This is an open-access article distributed under the terms of the Creative Commons Attribution License 3.0 (CC BY

3.0), which permits unrestricted use, distribution, and reproduction in any medium, provided the original author and source are credited.

\section{ABSTRACT}

In this study, we first initiated a multicenter, single-arm, phase-II clinical trial using decitabine (DAC) $\left(20 \mathrm{mg} / \mathrm{m}^{2}\right.$ for five days) based chemotherapy, followed by haploidentical lymphocyte infusion (HLI) that was applied as induction therapy for elderly patients with AML. Furthermore, the role of HLI infusion was explored in a mouse model. The clinical trial included 29 elderly patients (median age: 64, range 5777) with AML. Sixteen cases achieved complete remission (CR) and 9 cases achieved partial remission (PR) after the first treatment cycle. Of the patients with $P R, 5$ subjects achieved remission after the second induction, which brings the overall CR rate to $\mathbf{7 2 . 4 \%}$. The 2-year overall survival (OS) and disease-free survival (DFS) was $59.6 \%$ and $36.9 \%$ respectively. The treatment regimen was well tolerated with only one patient died of severe pneumonia one month after the first treatment. In the mouse experiment, we found that DAC/HLI significantly enhanced the survival of leukemic mice. These results suggested that DAC-based chemotherapy combined with HLI is an alternative first line induction therapy for elderly patients with AML. This trial is registered at ClinicalTrials.gov (NCT01690507).

\section{INTRODUCTION}

The majority of acute myeloid leukemia (AML) patients are older individual who are usually deemed ineligible for intensive chemotherapy. Up until now, the outcome of conventional induction chemotherapy for older patients with AML has been unsatisfactory. The two years OS in this group of patients is less than 10 percent [1]. Decitabine (DAC), a demethylating agent, was approved by European Union for the treatment of elderly patients with newly diagnosed AML who are not eligible for standard chemotherapy. As an epigenetic modulator, low dose DAC provides a promising approach for AML. However, less than 50 percent of older patients with AML achieved CR when DAC was administrated alone $[2,3]$. Recent studies showed that DAC can increase the chemosensitivity of leukemic [4] and several solid tumor cells [5], [6], [7]. Accumulating evidence also revealed that DAC may enhance the immunogenicity of malignant cells by inducing the expression of several 
immune-related genes including cancer testis antigen [8], major histocompatibility complex (MHC) molecules [9], costimulatory molecules [10] and adhesive molecules in these cells. Our previous study has demonstrated that DAC is capable of eliciting an anti-tumor CTL response by inducing CD80 expression in leukemic cells [11]. These two immunological effects of DAC offer it a great potential for developing DAC-based chemotherapy. However, the anti-leukemia activities of DAC-based chemotherapy could be limited by the immunocompromised status of AML patients due to chemotherapy and aging. Infusion of allogeneic lymphocytes following DAC-based therapy thus has the potential to overcome this limitation. Therefore, we hypothesized that adoptive transfer of haploidentical lymphocytes may act in synergy with DACbased chemotherapy for AML.

In this study, we first initiated a multicenter, single-arm, phase-II clinical trial using DAC (20mg/ $\mathrm{m}^{2}$ for five days)-based chemotherapy followed by infusion of haploidentical lymphocytes infusion (HLI) for the treatment of 29 elderly patients with AML. We found that DAC/HLI therapy was well tolerated and induced $\mathrm{CR}$ in $72.4 \%$ of AML patients. In an AML mice model, we further explored the role of HLI in the DAC/ HLI combined chemotherapy. We found that DAC/ HLI combination significantly enhanced the survival of leukemic mice. These results suggest that DAC-based chemotherapy combined with HLI is an alternative first line induction therapy for elderly patients with AML.

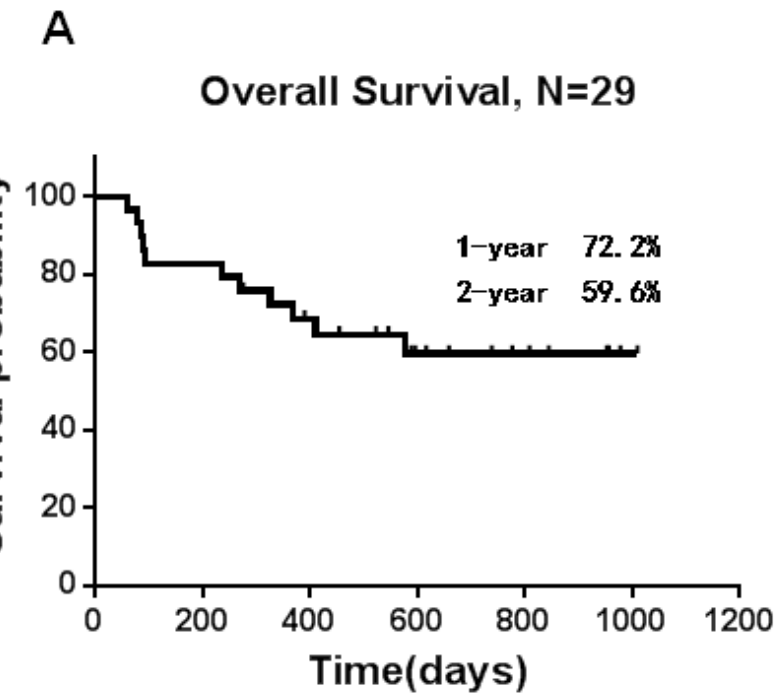

\section{RESULTS}

\section{HLI enhanced the survival of patients with leukemia receiving DAC-based chemotherapy}

DAC treatment causes cancer cells to express more tumor antigens and co-stimulatory molecules, thereby renders these cells more susceptible to T cell therapy. On the other hand, HLI has also been shown to be a promising method for the treatment of human leukemia [12]. Thus in this study, we designed a clinical trial protocol involving the use of DAC-based chemotherapy followed by HLI (ClinicalTrials.gov; NCT01690507). AML patients were assigned to receive chemotherapy with DAC $\left(20 \mathrm{mg} / \mathrm{m}^{2}\right.$ intravenously for 5 days), aclacinomycin (ACM, 20mg every second day intravenously for 5 days), cytarabine (10 mg/m² every 12 hours subcutaneously for 5 days), granulocyte colony-stimulating factor (G-CSF, $300 \mu \mathrm{g}$ / day subcutaneously from day 0 to neutrophil recovery), in combination with HLI treatment. HLI were performed 36 hours after the last dose of chemotherapy. Subjects who experienced $\mathrm{CR}$ or PR received a total of four repeated treatments. No maintenance treatment was given to patients who finished four treatments.

\section{Patient characteristics}

There were 29 patients with previously untreated AML defined by World Health Organization(WHO) criteria enrolled in the study, including 10 (34.5\%) women and $19(65.5 \%)$ men. The basic information of these patients is shown in Table 1. The median age of these patients was 64 (range 57-77), with 5 patients less than 60 , 14 patients with an age range of 60-69, and 6 patients over

B

\section{Disease Free Survival , $\mathrm{N}=21$}

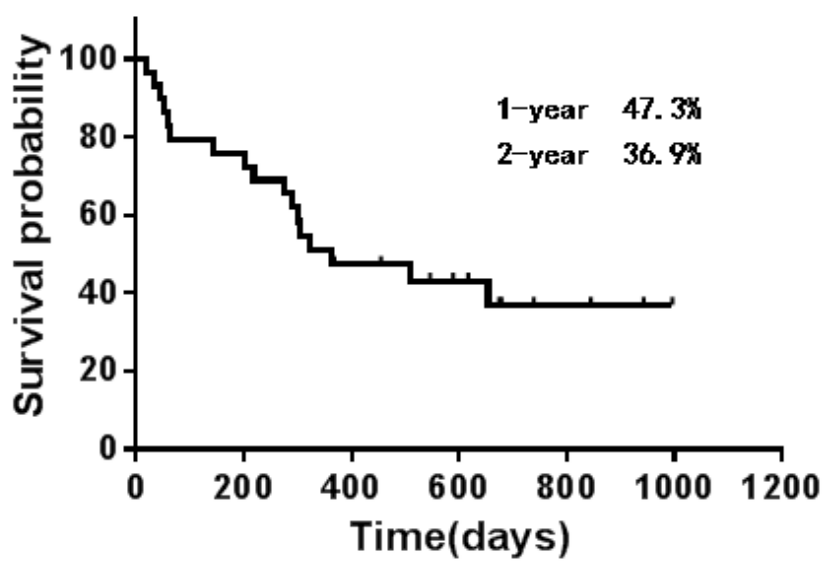

Figure 1: Survival results. A. Overall survival and B. disease-free survival. Vertical marks reflect last follow-up times for censored observations. 
Table 1: Patient characteristics

\begin{tabular}{|l|l|}
\hline & $\boldsymbol{n} \mathbf{( \% )}$ \\
\hline Gender & \\
\hline Men & $19(65.5)$ \\
\hline Women & $10(34.5)$ \\
\hline Diagnosis(WHO) & \\
\hline M1 & $0(0)$ \\
\hline M2 & $16(55.2)$ \\
\hline M4 & $4(13.8)$ \\
\hline M5 & $7(24.1)$ \\
\hline M6 & $2(6.9)$ \\
\hline Age(years) & \\
\hline $55-59$ & $5(17.2)$ \\
\hline $60-64$ & $14(48.3)$ \\
\hline $65-69$ & $4(13.8)$ \\
\hline$\geq 70$ & $6(20.7)$ \\
\hline Performance status(ECOG sore) & \\
\hline 1 & $5(17.2)$ \\
\hline 2 & $12(41.4)$ \\
\hline 3 & $12(41.4)$ \\
\hline WBC at diagnosis,10×10/L & \\
\hline$\leq 20$ & $22(75.9)$ \\
\hline$>20-100$ & $4(13.8)$ \\
\hline$\geq 100$ & $3(10.3)$ \\
\hline Cytogenetic risk & \\
\hline Favorable & $0(0)$ \\
\hline Intermediate & $20(69)$ \\
\hline Unfavorable & $7(24.1)$ \\
\hline unavilable & $2(6.9)$ \\
\hline Comorbid conditions & \\
\hline Lung Infection & $4(13.8)$ \\
\hline Diabetes & $6(20.7)$ \\
\hline Hypertension & $12(41.4)$ \\
\hline Cardiovascular disease & $6(20.7)$ \\
\hline Others & $10(34.5)$ \\
\hline & \\
\hline & \\
\hline
\end{tabular}

70,8 subjects $(28 \%)$ had normal karyotype, 6 subjects $(30 \%)$ complex karyotype, and 15 other cytogenetic abnormalities.

According to the validated Medical Research Council (MRC) prognostic risk score for clinical outcome in older AML patients [13], 27.5\% of enrollees were in poor risk, $41.5 \%$ in standard risk, and $31 \%$ in good risk.

Twenty four subjects $(82.75 \%)$ had a performance status of $\geq 2$. The median white blood cell(WBC) count was $25.7 \times 10^{9} / \mathrm{L}$ (range $0.1-270 \times 10^{9} / \mathrm{L}$ ). The median bone marrow blast count was $60 \%(20.6 \%-95 \%)$. Twenty two subjects had an intermediate and seven subjects (24\%) were with an unfavorable cytogenetic risk. Two subjects (5\%) had the MLL/MLL or MLL/AF9 fusion gene and three subjects $(10 \%)$ had a WBC more than $100 \times 10^{9} / \mathrm{L}$. Seven subjects received cytarabine treatment due to the fact that their $\mathrm{WBC}$ were more than $20 \times 10^{9} / \mathrm{L}$ before the initiation of DAC-based chemotherapy. Cytarabine was given to the patients at $100 \mathrm{mg}$ to $200 \mathrm{mg}$ intravenously each day, and was stopped when WBC was lower than $10 \times 10^{9} / \mathrm{L}$. The DAC/HLI treatment was started at the next day after the cessation of cytarabine. The median followup was 18.2 months (range 2-33.7 months).

\section{Efficacy of HLI and DAC-based combination therapy}

Of 29 patients receiving combination treatment with DAC-based chemotherapy and HLI, 16 achieved CR and 9 achieved partial PR after the first treatment cycle. Of the patients with PR, 5 subjects achieved CR after the second induction, which brings the overall CR rate to $72.4 \%$. CR occurred in all risk groups. For subjects with good risk, $88.9 \%$ (8 of 9) achieved CR. For subjects with standard risk, $66.7 \%$ (8 of 12) achieved CR. For subjects with poor risk, $62.5 \%$ (5 of 8 ) achieved CR. 
Table 2: Treatment-Emergent Grades III/IV AEs during the first two cycles

\begin{tabular}{|l|l|l|}
\hline AEs of $\geq$ Grade III & No. & Percentage \\
\hline Atrial fibrilation & $1 / 29$ & $3.4 \%$ \\
\hline Blood bilirubin increased & $1 / 29$ & $3.4 \%$ \\
\hline Diarrhea & $1 / 29$ & $3.4 \%$ \\
\hline Febrile neutropenia & $16 / 29$ & $55.2 \%$ \\
\hline Gastrointestinal hemorrhage & $1 / 29$ & $3.4 \%$ \\
\hline Heart failure & $1 / 29$ & $3.4 \%$ \\
\hline Lung infection & $2 / 29$ & $6.8 \%$ \\
\hline Rash & $1 / 29$ & $3.4 \%$ \\
\hline
\end{tabular}

Upon final analysis (May 31, 2015), the median survival time was 546 days for all 29 subjects. The 2-year probability of OS (Figure 1A) and Disease-free survival (DFS) (Figure 1B) was 59.6 $\pm 9.6 \%$ and $36.9 \pm 9.9 \%$, respectively. The 1-year probability of OS and DFS of patients received prior cytarabine therapy due to hyperleukocytosis were $42.9 \pm 18.7 \%$ and $14.3 \pm 13.2 \%$, respectively. For patients with no prior therapy, The 1-year probability of OS and DFS was $76.4 \pm 9.3 \%$ and $50.9 \pm 11.3 \%$, respectively. For subjects with good risk, the 1 -year probability of OS and DFS was $64.8 \pm 16.5 \%$ and $41.5 \pm 20.7 \%$, respectively. For subjects with standard risk, the 1-year probability of OS and DFS was $83.3 \pm 10.8 \%$ and $58.3 \pm 14.2 \%$, respectively. For subjects with poor risk, the 1-year probability of OS and DFS was $37.5 \% \pm 17.1 \%$ and $25 \pm 15.3 \%$, respectively. 21 patients achieved CR after two cycle of treatment, the 1-year probability of OS and DFS was $80.4 \pm 8.8 \%$ and $54.5 \pm 11.3 \%$, respectively. There were only 2 patients who achieved partial remission(PR) after two cycle of treatment. One patient refused further treatment and died within 90 days. The other patient received best supportive care and traditional Chinese medicine treatment and died 411 days after diagnosis. There were limited data about the patients with molecular markers. One patient with FLT3-ITD did not achieve CR and died of disease progress within 3 months. One patient with MLL/AF9 achieved molecular remission after 2 cycles of treatment and received 2 cycles of consolidation treatment with the same scheme of induction. The patient relapsed 4 months after the last treatment. She could not achieve CR and died within 3 months. One patient with MLL/MLL achieved morphologic CR and the ratio of fusion gene/ABL decreased from $26.3 \%$ to $4.3 \%$ after 2 cycle of treatment. He received additional 2 cycle of consolidation treatment with the same regimen but failed to test the fusion gene. He relapsed 6 months later and received chemotherapy with mitoxantrone and cytarabine. $\mathrm{CR}$ was achieved again. He remained CR up until the last follow-up.

Thirteen subjects (44.8\%) died during the followup. Of those, three subjects did not achieve CR and died of disease progression and 6 subjects died of leukemia relapse, 2 subjects died of pneumonia, including one patient died at 37 days after chemotherapy, and 2 subjects died of gastrointestinal hemorrhage.

\section{Safety and toxicity}

Overall, the combination regimen was well tolerated. The median recovery times for neutrophils and platelets were 11 days and 14 days, respectively, after the first cycle of chemotherapy. The median number of $\mathrm{WBC}$ and platelets were more than $1.0 \times 10^{9} / \mathrm{L}$ (Figure $2 \mathrm{~A}$ ) and $20 \times 10^{9} / \mathrm{L}$ (Figure $2 \mathrm{~B}$ ), respectively, after the first cycle of chemotherapy induction. Three patients did not exhibit neutropenia. The platelet counts of 2 patients kept higher than $20 \times 10^{9} / \mathrm{L}$ during the whole treatment. Myelosuppression was commonly observed, and febrile neutropenia occurred in $55.2 \%$ of patients. No subjects needed to be transferred to Intensive Care Unit.

All patients were evaluated for toxicity. The most common grade 3 or 4 adverse events (AE) are listed in Table 2. Infectious complications were rare following achievement of CR. Myelosuppression during maintenance treatment given after CR was minimal.

\section{GVHD and severe infection}

No symptoms of acute or chronic Graft Versus Host Disease (GVHD), such as unexplained skin rashes, diarrhea, increased bilirubin or disturbance of coagulation, was observed in any of the subjects during treatment. Four subjects suffered from pneumonia during the first 2 cycles of chemotherapy. Two subjects were diagnosed as pulmonary fungal infection between the second and third 
cycle of chemotherapy and were controlled with antifungal agents.

\section{HLI enhanced the survival of mice with leukemia receiving DAC-based chemotherapy}

The results of our clinical trial showed that the DAC-based chemotherapy combined with HLI achieved higher CR rate than that of intensive chemotherapy. We hypothesized that HLI may act synergistically with DACbased chemotherapy in eradicating leukemic cells. To test this hypothesis, we conducted a series of experiments using a mouse AML model. BALB/c mice were injected with mouse leukemia WEHI-3 cells i.p. and treated with DAC and cytarabine(Ara-C) with or without HLI. As shown in Figure 3A, while DAC + Ara-C combination showed significant efficacy in promoting the survival of mice with leukemia, HLI further improved the survival of mice treated with $\mathrm{DAC}+\mathrm{Ara}-\mathrm{C}$ combination. These data suggested that HLI enhances the efficacy of DAC+Ara-C chemotherapy, leading to longer survival of mice with leukemia.

While the detailed mechanisms of HLI-mediated survival benefit remain elusive, We have previously demonstrated that DAC treatment induces anti-tumor CTL responses in a mouse solid tumor model. Herein we hypothesized that DAC treatment could render tumor cells more susceptible to the destruction induced by lymphocytes such as CTLs; Furthermore, DAC has the

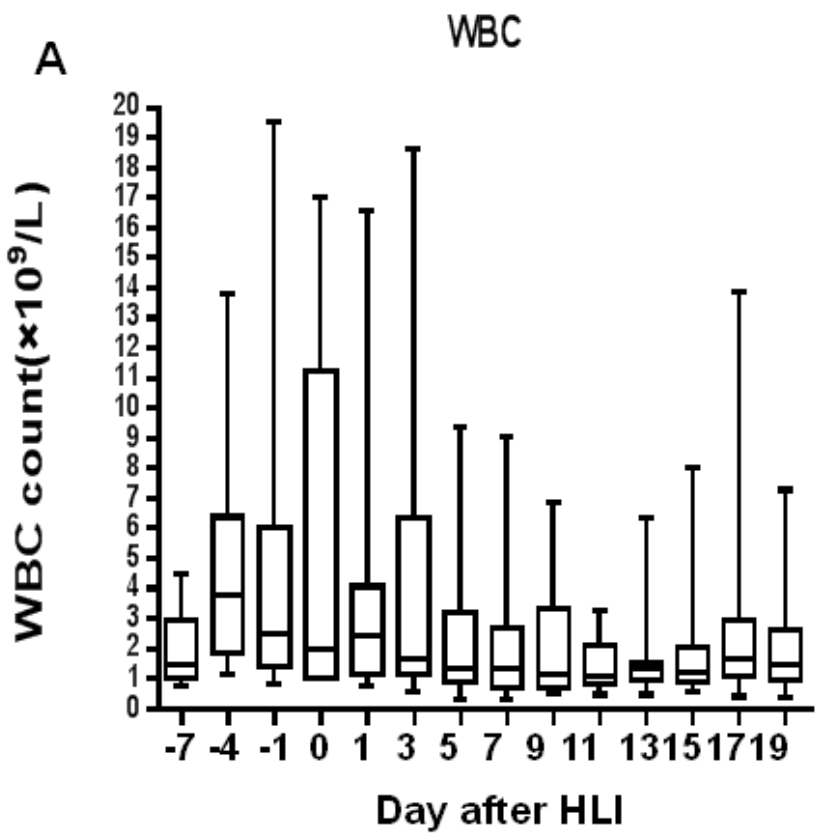

potential to enhance the priming of $\mathrm{T}$ cell responses, which may act in synergistic with adoptive transfer of HLI. To test the effect of DAC treatment on CTL response in mice with leukemia, BALB/c mice with leukemia were treated with PBS or DAC. Seven days later mice were sacrificed and spleen cells were harvested. CD3+ cells were purified using flow cytometry-based sorting followed by infusion into $\mathrm{BALB} / \mathrm{c}$ nude mice. The nude mice were then challenged with DAC-treated WEHI-3 cells. As shown in Figure $3 \mathrm{~B}$, mice receiving $\mathrm{T}$ cells from DAC-treated mice experienced significantly longer survival compared with mice treated with $\mathrm{T}$ cells from control mice.

\section{DISCUSSION}

In this study, we first initiated a multicenter, singlearm, phase-II clinical trial using DAC/HLI to treat 29 elderly patients with AML. We found that DAC/HLI therapy was well-tolerated and induced CR in a total of $72.4 \%$ of AML patients after two cycles of treatments. Notably, 6 out of $29(20.6 \%)$ patients treated in this study were older than 70 years old, and $24(86.7 \%)$ had ECOG Performance Status (ECOG PS) $\geq 2$. Furthermore, we conducted a series of experiments to investigate the role of HLI in an AML mouse model. We found that mice received HLI containing therapy had a significant longer survival than mice received chemotherapy alone, which suggested HLI exerts a synergistic effect with DAC-based chemotherapy. In addition, we revealed DAC treatment elicited autologous CTL response against AML.

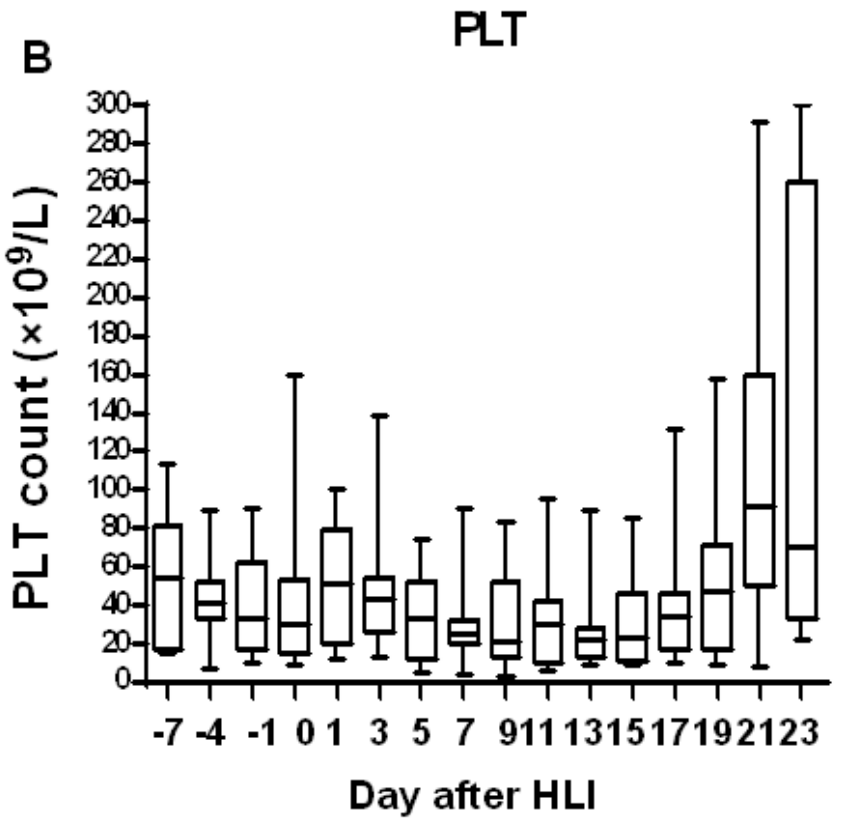

Figure 2: Blood count recovery after induction therapy of DAC-based chemotherapy combined with haplo-identical donor lymphocytes. Box plots show the median (line), interquartile (box extents), and range (whiskers) of WBC A. and platelet count B. for all patients during the time of the induction therapy. Blood count data are summarized every second day. 
For the last two decades, little progress has been made in the treatment of elderly patients with AML and there remains no consensus regarding optimal therapy. The outcome of the intensive chemotherapy in elderly patients with AML has been unsatisfying. Generally, the CR rate was about $40-55 \%$ and the early death (ED) rate was 19 $29 \%$ [14].

Low dose cytarabine, aclaribicin and G-CSF (CAG) regimen was originally designed in Japan for the treatment of relapsed AML patients [15]. Several clinical studies showed CAG regimen achieved about $50 \%$ CR in elderly patients with AML with lower early death(about 8\%) [16, 17]. In a recent study, single-agent DAC was conducted in older patients ( $\geq 60$ years) with previously untreated AML who were not candidates for or refused intensive chemotherapy. The complete remission rate was $47 \%$ ( $n$
$=25$ ), achieved after a median of three cycles of therapy. Death within 8 weeks occurred in $15 \%$ of subjects [3]. The results of our clinical trial showed that DAC/HLI was well tolerated and effective with CR of $72.4 \%$ in elderly patients with AML after two cycle of treatment, while only 1 patient $(3.4 \%)$ died within 8 weeks. A recent study reported a prospective phase II clinical trial assessing the safety and efficacy of D-CAG (DAC combined with cytarabine, aclarubicin, and G-CSF) induction treatment for elderly patients with newly diagnosed AML [18]. Among 85 evaluable patients, CR was $64.7 \%$ after 1 cycle of therapy. The induction mortality was $4.4 \%$. According to their report, $77.6 \%$ patients achieved $\mathrm{CR}$ after two cycles of treatment. The CR rate of their regimen is comparable to the data presented here. While agents in their treatment were the same as ours, the
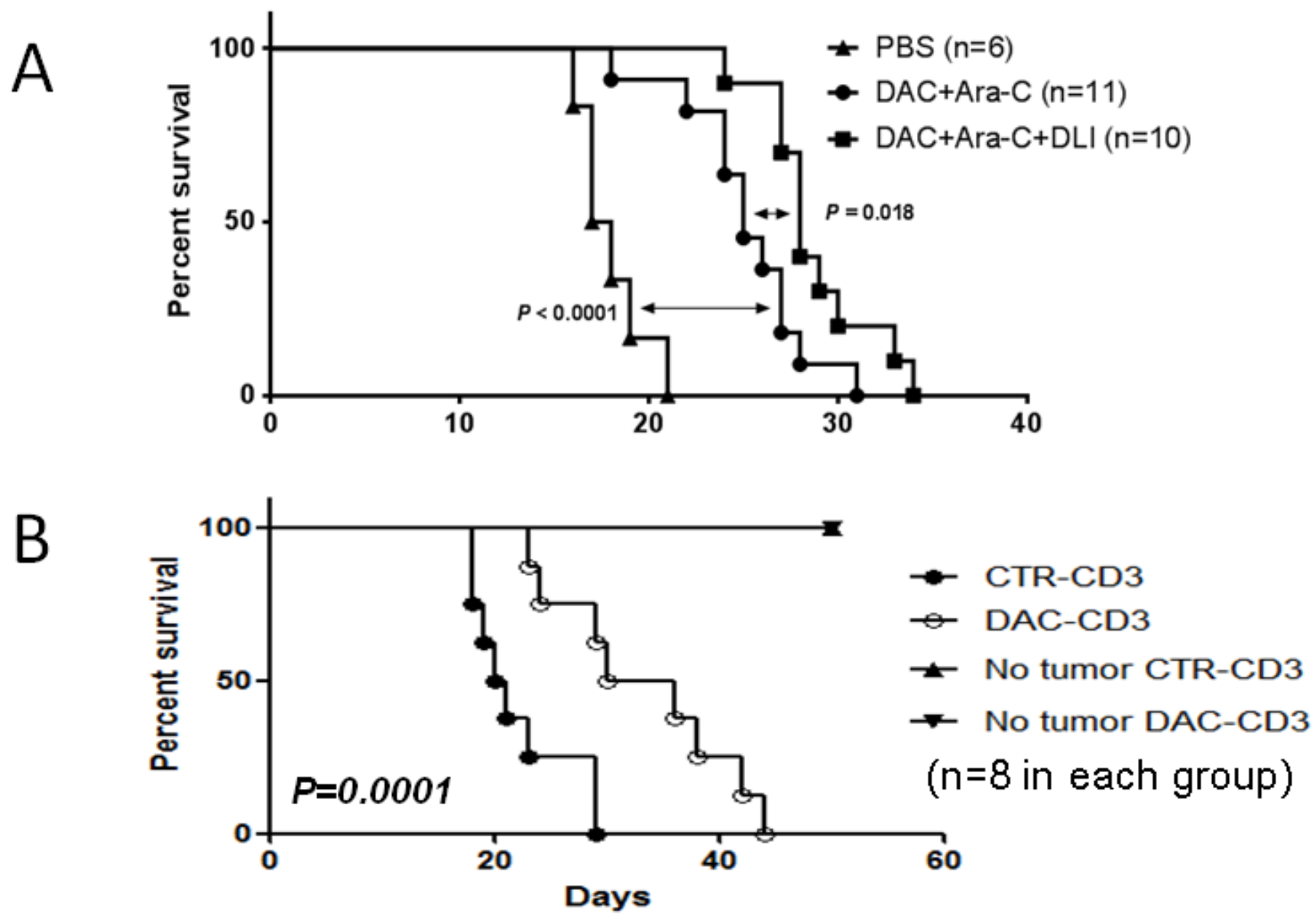

Figure 3: DAC treatment induced autologous anti-leukemia response and acted in synergy with adoptive $T$ cells transfusion. A. WEHI-3 cells were injected i.p. into Balb/c mice at $1 \times 10^{5}$ cells $/$ mouse. DAC $(0.5 \mathrm{mg} / \mathrm{kg} / \mathrm{day}$ for 5 days $)$ and cytarabine $(10 \mathrm{mg} / \mathrm{kg} / \mathrm{d}$ for 5 days) were administrated 5 days later. Lymphocyte from CBF1 mice were injected intravenously 48 hours later. Mouse survival was monitored every two or three days. Five to six mice were used in each group and data were pooled from two experiments. B. WEHI-3 cells were injected i.p. into Balb/c mice at $1 \times 10^{5}$ cells $/$ mouse. DAC $(1 \mathrm{mg} / \mathrm{kg} /$ day for 3 days $)$ or PBS were administrated 5 days later. Purified $\mathrm{CD}^{+}$cells were harvested from each mouse 7 days after the treatment for transfer. Meanwhile, in vitro DAC $(0.25 \mathrm{uM}$ for 3 days) treated WEHI3 cells were injected into naïve Balb/c nude mice i.p. at $5 \times 10^{4}$ cells $/$ mouse. Then harvested CD3 ${ }^{+}$cells were adoptively transferred intravenously into with/without WEHI3 nude mice at $2 \times 10^{7}$ cells/mouse. Mouse survival data is shown. Eight mice were included in each group and data shown are representative of two independent experiments. 
duration and dosage of cytarabine and aclarubicin were different. The most significant difference between the two regimens was that HLI was included in ours at day seven. It is difficult to evaluate the role of HLI directly because of different conditions and settings in these two studies. Our animal experiments demonstrated that HLI exerted synergistic effects with previous DAC-based chemotherapy. Apparently, prospective, randomized and control studies are needed to investigate this aspect. In addition, we believed there still is a plenty of room for us to improve the combination treatment by optimizing the cell components.

In this clinical study, 7 patients received prior cytarabine due to the fact that there was a high number of circulating peripheral blasts or that they were even hyperleukocytair. Given cytarabine is the cornerstone of an AML induction therapy, we compared the OS and DFS of patients who received prior cytarabine therapy with those who did not receive prior therapy. We found that prior cytarabine therapy did not contribute to longer OS or DFS. On the other hand, hyperleukocytair might play a central role in the survival of these patients.

The results of our clinical trial demonstrated that the combination treatment was well tolerated for elderly patients, who were traditionally deemed as unfit intensive chemotherapy. There was only one patient died within the first 8 weeks. The degree of myelosuppresion was relatively lower than that after intensive regimen. The duration of neutropenia was only 11 days. We speculated that HLI may play an important role to enhance the immune recovery after chemotherapy and to reduce the risk of infections.

In the study, 6 out of 21 patients who achieved CR experienced relapse within median duration of 8.4 months(5.9-21.6 months). Among these 6 patients, 3 were in poor risk, 1 in standard risk, and 2 in good risk. For elderly patients with AML, there is no consensus postremission treatment available [19]. Additional 2 or 3 cycles of treatment were given to the patients after they achieved $\mathrm{CR}$ in our study. The relapse rate suggested that more maintenance treatment should be considered for these patients.

In conclusion, our study demonstrated that DACbased chemotherapy followed by HLI is a safe and effective induction regimen for untreated elderly patients with AML. The results suggested that DAC/HLI may significantly improve responding rate compared with other chemotherapy regimens those are currently using. Further a randomized-controlled trial in a large cohort of subjects is required to confirm the safety and efficacy of this combination therapy. The underlying therapeutic mechanisms, in particular the role of HLI, also warrant further investigations.

\section{MATERIALS AND METHODS}

\section{Patients}

Twenty nine patients with newly diagnosed AML (from April, 2012 to May, 2015) were enrolled in this study. The eligibility criteria were age over 55 years with previously untreated AML (marrow blasts $\geq 20 \%$, de novo or secondary to myelodysplastic syndrome). The diagnoses were defined by the French-American-British and World Health Organization criteria. Chromosomal analysis and immunophenotyping were performed for all subjects using bone marrows obtained at diagnosis.

High resolution HLA-typing was conducted in all subjects and donors. Sex, age, and other characteristics of lymphocyte donors were not considered as a priority. Of the 29 patient/donor pairs, the median age of donors was 34 (range 24-54), including 15 sons, 12 daughters, 1 younger brothers and 1 younger sister.

The protocol was approved by the Human Ethics Committees of the Chinese PLA General Hospital, Beijing; Navy General Hospital of PLA, Beijing; the First Affilliated Hospital of Dalian Medical University, Liaoning province; and Dalian Municipal Central Hospital Affilliated to Dalian Medical University, Liaoning Province, China. This protocol was conducted in accordance with the Declaration of Helsinki. All patients and donors gave written informed consent before enrollment into the study. This trial was registered at www.clinicaltrials.gov as NCT01690507.

\section{Treatment design}

Subjects were assigned to receive chemotherapy with DAC (20 $\mathrm{mg} / \mathrm{m}^{2}$ intravenously for 5 days), aclacinomycin (ACM, 20mg every second day intravenously for 5 days $)$, cytarabine $\left(10 \mathrm{mg} / \mathrm{m}^{2}\right.$ every 12 hours subcutaneously for 5 days), granulocyte colonystimulating factor (G-CSF, $300 \mu \mathrm{g} /$ day subcutaneously from day 0 to neutrophil recovery) every 4 weeks. The subsequent cycles was individually customized based on the duration of neutropenia. HLI were administered 36 hours after the final dose of chemotherapy. For the first induction therapy, the cytarabine was given to the patients with white blood cell(WBC) count higher than $20 \times 10^{9} / \mathrm{L}$ at $100 \mathrm{mg}$ to $200 \mathrm{mg}$ intravenously for each day, and was stopped when $\mathrm{WBC}$ was lower than $10 \times 10^{9} / \mathrm{L}$. The DAC/HLI treatment was started at the next day after the cessation of cytarabine. Subjects who experienced CR or PR received a total of four courses of the same treatment. No maintenance treatment was given to patients who finished four treatments.

Routine blood counts, electrolyte levels, liver function tests, and creatinine levels were monitored 
twice a week. Adverse events, concomitant medications, and clinical laboratory analyses were recorded weekly. The treatment was discontinued in cases of disease progression, intolerable toxicity, death, losses of followup, treatment abandonment, or withdrawal of consent to further treatment. All subjects received supportive care, including blood product transfusions, prophylactic or symptomatic use of antibiotic agents and cytokines, according to institutional practices and other therapies appropriate for the symptomatic treatment for AML and its complications.

Subjects who did not achieve CR after two cycles of chemotherapy, or those who experienced relapses during the first two cycles were considered as treatment failure and treatment was discontinued. None of the subjects received any GVHD prophylactic treatment or further maintenance therapy.

\section{Apheresis of donor peripheral mononuclear cells}

Apheresis of haplotype-identical donor peripheral mononuclear cells was carried out with a cell separator (COBE SPECTRA cell separator). Donor cells were divided into aliquots and were cryopreserved in liquid nitrogen, whereas freshly collected cells were used in the first cycle. Infusion was performed at 36 hours after each chemotherapy regimen. The median numbers of mononuclear cells, $\mathrm{CD}^{+}, \mathrm{CD}^{-} \mathrm{CD}^{-} 9^{+}$, and $\mathrm{CD}^{-}$ $\mathrm{CD} 16^{+} \mathrm{CD}^{2} 6^{+}$cells infused per treatment were $1.51 \times 10^{8} /$ $\mathrm{kg}\left(0.77-2.57 \times 10^{8} / \mathrm{kg}\right), 0.935 \times 10^{8} / \mathrm{kg}\left(0.62-2.03 \times 10^{8} / \mathrm{kg}\right)$, $0.18 \times 10^{8} / \mathrm{kg}\left(0.048-0.3 \times 10^{8} / \mathrm{kg}\right)$ and $0.089 \times 10^{8} / \mathrm{kg}(0.041-$ $\left.0.23 \times 10^{8} / \mathrm{kg}\right)$, respectively.

\section{Response criteria and outcome evaluation}

Routine blood cell counts were performed twice per week after chemotherapy. At three to four weeks after chemotherapy, a bone marrow aspiration was performed and responses to treatment were evaluated. Responses were determined according to the revised recommendations of the International Working Group (IWG) for Diagnosis, Standardization of Response Criteria, Treatment Outcomes, and Reporting Standards for Therapeutic Trials in Acute Myeloid Leukemia. OS was defined as the time from diagnosis to death or to the last date of follow-up until May 2015. For secondary end points, bone marrow biopsies and aspirates were obtained from subjects at screening.

The following conditions were defined according to the IWG criteria: (1) CR was diagnosed for subjects demonstrating less than 5\% bone marrow blasts, no blasts with Auer rods, the absence of extramedullary disease, an absolute neutrophil count $>1 \times 10^{9} / \mathrm{L}$, and a platelet count $\geq 100 \times 10^{9} / \mathrm{L}$; (2) PR was assigned to subjects with a decrease of at least 50\% (between 5 and 20\%) in the frequency of blasts detected in bone marrow aspirates and those with normalized blood counts; (3)No remission(NR) was defined as a response that had achieved neither CR nor PR; and (4) relapse was defined as the reappearance of leukemia cells in the peripheral blood or the detection of more than $5 \%$ blasts in the bone marrow. Early death was defined as mortality within the first 8 weeks after induction of chemotherapy. GVHD was defined according to published criteria [20].

The recovery time of neutrophils and platelets was defined as the first of 3 consecutive days on which the absolute neutrophil count and platelet count exceeded $0.5 \times 10^{9} / \mathrm{L}$ and $30 \times 10^{9} / \mathrm{L}$, respectively, since the day of HLI. Adverse events were determined according to the CTCAE version 4.03 .

\section{Mice and cells}

$\mathrm{Balb} / \mathrm{c}$ and Balb/c nude mice (male, 6-8 week) were purchased from Beijing Vital-River Lab Animal Technology Co. Ltd. WEHI-3 (a mouse AML cell line) was originally obtained from American Type Culture Collection (ATCC). WEHI-3 cells were cultured and maintained in Dulbecco's Modified Eagle Medium (DMEM) supplemented with 10\% heat-inactivated equine serum (Hyclone), $100 \mu \mathrm{g} / \mathrm{ml}$ penicillin/streptomycin and L-glutamine. The cells were incubated at $37{ }^{\circ} \mathrm{C}$ in $5 \%$ $\mathrm{CO}_{2}$. All mice were maintained at Chinese PLA General Hospital (CPGH) in specific pathogen-free conditions. All animal experiments were performed in accordance with national and institutional guidelines for animal care and approved by the animal use and care committee, CPGH.

\section{DAC combined with cytarabine and lymphocyte infusion for mice with AML}

Balb/c mice were injected i.p. with WEHI-3 cells $\left(10^{5} /\right.$ mouse) and then randomly assigned into three groups. Group one was treated with PBS. Group two and Group three were injected i.p. with DAC $(0.5 \mathrm{mg} / \mathrm{kg} / \mathrm{d})$ and cytarabine $(10 \mathrm{mg} / \mathrm{kg} / \mathrm{d})$ for 5 consecutive days. For group three, splenocytes from naïve C $57 \times$ Balb/c F1 (CB6F1) mice were injected i.v. at a dose of $4 \times 10^{7} /$ mouse 36 hours after DAC treatment. The survival of mice was observed every 2 or 3 days.

\section{Adoptive transfer of $\mathrm{CD3}^{+}$splenocytes from DAC treated mice}

For leukemia establishment, Balb/c mice were injected i.p. with WEHI-3 cells ( $10^{5} /$ mouse). The mice were injected with either PBS or DAC $(0.5 \mathrm{mg} / \mathrm{kg} / \mathrm{d})$ i.p. once per day for 5 days. Treated mice were sacrificed seven days later for lymphocyte harvesting. Following red 
blood cell lysis procedure, splenocytes were labeled with PE-conjugated anti-mouse CD3 mAb and consequently anti-PE microbeads (Miltenyi), followed by MACS selection. Purity of positively selected spleen $\mathrm{CD}^{+}$cells was determined by FACS analysis. Purified CD3 positive cells $\left(2 \times 10^{7}\right)$ from PBS or DAC treated mice were injected i.v. into $\mathrm{Balb} / \mathrm{C}$ nude mice, which were injected with/ without DAC-treated (1 uM for three days in culture) WEHI- 3 cells $\left(5 \times 10^{4}\right.$ cells/mouse $)$ two days earlier. The survival of mice was observed every 2 or 3 days.

\section{Statistical analysis}

SAS 9.0 software (SAS Institute) was used in all statistical analysis. Survival data were analyzed by the log-rank test, and survival curves were plotted with the Kaplan-Meier method. $t$ test was used to assess the probability of significant differences of survival time. $P<$ 0.05 was considered statistically significant.

\section{CONFLICTS OF INTEREST}

The authors declare that there is no conflict of interest.

\section{GRANT SUPPORT}

This work was supported by grants from the National Natural Science Foundation of China (81170518, 81270611, 81370635, 81350004 and 81470010), Capital Medical Development Scientific Research Fund (SF20015001-07), National Public Health Grant Research Foundation (No.201202017) and The capital of the public health project (Z111107067311070), and Inovation and Fostoring Foundation of China PLA Navy General Hospital (cxpy201302).

\section{REFERENCES}

1. Pollyea DA, Kohrt HE, Medeiros BC. Acute myeloid leukaemia in the elderly: a review. British journal of haematology. 2011; 152:524-542.

2. Lubbert M, Suciu S, Baila L, Ruter BH, Platzbecker U, Giagounidis A, Selleslag D, Labar B, Germing U, Salih HR, Beeldens F, Muus P, Pfluger KH, et al. Lowdose decitabine versus best supportive care in elderly patients with intermediate- or high-risk myelodysplastic syndrome (MDS) ineligible for intensive chemotherapy: final results of the randomized phase III study of the European Organisation for Research and Treatment of Cancer Leukemia Group and the German MDS Study Group. Journal of clinical oncology : official journal of the American Society of Clinical Oncology. 2011; 29:19871996.
3. Blum W, Garzon R, Klisovic RB, Schwind S, Walker A, Geyer S, Liu S, Havelange V, Becker H, Schaaf L, Mickle J, Devine H, Kefauver C, et al. Clinical response and miR$29 \mathrm{~b}$ predictive significance in older AML patients treated with a 10-day schedule of decitabine. Proceedings of the National Academy of Sciences of the United States of America. 2010; 107:7473-7478.

4. Bhatla T, Wang J, Morrison DJ, Raetz EA, Burke MJ, Brown P, Carroll WL. Epigenetic reprogramming reverses the relapse-specific gene expression signature and restores chemosensitivity in childhood B-lymphoblastic leukemia. Blood. 2012; 119:5201-5210.

5. Momparler RL. Epigenetic therapy of non-small cell lung cancer using decitabine (5-aza-2'-deoxycytidine). Frontiers in oncology. 2013; 3:188.

6. Fang F, Zuo Q, Pilrose J, Wang Y, Shen C, Li M, Wulfridge P, Matei D, Nephew KP. Decitabine reactivated pathways in platinum resistant ovarian cancer. Oncotarget. 2014; 5:3579-3589. doi: 10.18632/oncotarget.1961.

7. Krishnadas DK, Shapiro T, Lucas K. Complete remission following decitabine/dendritic cell vaccine for relapsed neuroblastoma. Pediatrics. 2013; 131:e336-341.

8. Zhang W, Barger C, Link P, Mhawech-Fauceglia P, Miller A, Akers S, Odunsi K, Karpf AR. DNA hypomethylationmediated activation of Cancer/Testis Antigen 45 (CT45) genes is associated with disease progression and reduced survival in epithelial ovarian cancer. Epigenetics : official journal of the DNA Methylation Society. 2015: 1062206.

9. Wastowski IJ, Simoes RT, Yaghi L, Donadi EA, Pancoto JT, Poras I, Lechapt-Zalcman E, Bernaudin M, Valable S, Carlotti CG Jr, Flajollet S, Jensen SS, Ferrone S, et al. Human leukocyte antigen-G is frequently expressed in glioblastoma and may be induced in vitro by combined 5-aza-2'-deoxycytidine and interferon-gamma treatments: results from a multicentric study. The American journal of pathology. 2013; 182:540-552.

10. Coral S, Sigalotti L, Gasparollo A, Cattarossi I, Visintin A, Cattelan A, Altomonte M, Maio M. Prolonged upregulation of the expression of HLA class I antigens and costimulatory molecules on melanoma cells treated with 5-aza-2'deoxycytidine (5-AZA-CdR). Journal of immunotherapy. 1999; 22:16-24.

11. Wang LX, Mei ZY, Zhou JH, Yao YS, Li YH, Xu YH, Li JX, Gao XN, Zhou MH, Jiang MM, Gao L, Ding Y, $\mathrm{Lu} \mathrm{XC}$, et al. Low dose decitabine treatment induces CD80 expression in cancer cells and stimulates tumor specific cytotoxic T lymphocyte responses. PloS one. 2013; 8:e62924.

12. Guo M, Hu KX, Yu CL, Sun QY, Qiao JH, Wang DH, Liu GX, Sun WJ, Wei L, Sun XD, Huang YJ, Qiao JX, Dong Z, et al. Infusion of HLA-mismatched peripheral blood stem cells improves the outcome of chemotherapy for acute myeloid leukemia in elderly patients. Blood. 2011; 117:936-941. 
13. Wheatley K, Brookes CL, Howman AJ, Goldstone AH, Milligan DW, Prentice AG, Moorman AV, Burnett AK; United Kingdom National Cancer Research Institute Haematological Oncology Clinical Studies G, Acute Myeloid Leukaemia S. Prognostic factor analysis of the survival of elderly patients with AML in the MRC AML11 and LRF AML14 trials. British journal of haematology. 2009; 145:598-605.

14. Ossenkoppele G, Lowenberg B. How I treat the older patient with acute myeloid leukemia. Blood. 2015; 125:767774.

15. Yamada K, Furusawa S, Saito K, Waga K, Koike T, Arimura H, Aoyagi A, Yamato H, Sakuma H, Tsunogake $\mathrm{S}$, et al. Concurrent use of granulocyte colony-stimulating factor with low-dose cytosine arabinoside and aclarubicin for previously treated acute myelogenous leukemia: a pilot study. Leukemia. 1995; 9:10-14.

16. Qian SX, Li JY, Tian T, Shen YF, Jiang YQ, Lu H, Wu HX, Zhang SJ, Xu W. Effect of low-dose cytarabine and aclarubicin in combination with granulocyte colonystimulating factor priming (CAG regimen) on the outcome of elderly patients with acute myeloid leukemia. Leukemia research. 2007; 31:1383-1388.

17. Suzushima H, Wada N, Yamasaki H, Eto K, Shimomura
T, Kugimiya MH, Horikawa K, Nishimura S, Tsuda H, Mitsuya H, Asou N. Low-dose cytarabine and aclarubicin in combination with granulocyte colony-stimulating factor for elderly patients with previously untreated acute myeloid leukemia. Leukemia research. 2010; 34:610-614.

18. Li J, Chen Y, Zhu Y, Zhou J, Xu Y, Li Y, Yu K, Pan L, Wang J, Ding J, Gu J, Zhou S, Shi J, et al. Efficacy and safety of decitabine in combination with G-CSF, low-dose cytarabine and aclarubicin in newly diagnosed elderly patients with acute myeloid leukemia. Oncotarget. 2015; 6:6448-6458. doi: 10.18632/oncotarget.3361.

19. Yanada M, Naoe T. Acute myeloid leukemia in older adults. International journal of hematology. 2012; 96:186-193.

20. Przepiorka D, Weisdorf D, Martin P, Klingemann HG, Beatty P, Hows J, Thomas ED. 1994 Consensus Conference on Acute GVHD Grading. Bone marrow transplantation. $1995 ; 15: 825-828$. 